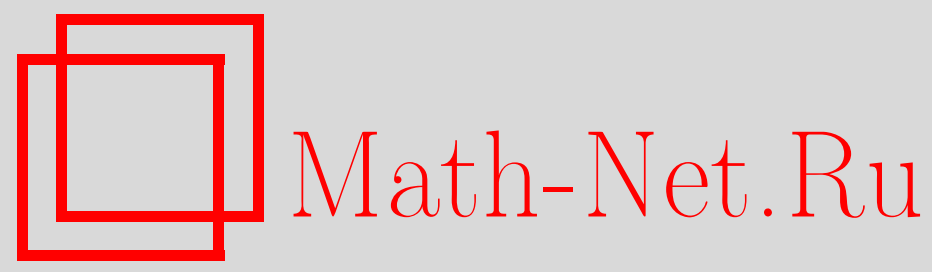

С. А. Волков, Конечная порождаемость некоторых групп рекурсивных перестановок, Дискрет. матем., 2008, том 20, выпуск 4, 61-78

DOI: https://doi.org/10.4213/dm1026

Использование Общероссийского математического портала Math-Net.Ru подразумевает, что вы прочитали и согласны с пользовательским соглашением http://www . mathnet.ru/rus/agreement

Параметры загрузки:

IP : 3.89 .185 .249

26 апреля 2023 г., 09:52:02 


\title{
Конечная порождаемость некоторых групп рекурсивных перестановок
}

\author{
(ㄷ) 2008 г. С. А. Волков
}

\begin{abstract}
Пусть класс $Q$ функций натурального аргумента замкнут относительно суперпозиции и содержит тождественную функцию. Множество перестановок $f$ таких, что $f, f^{-1} \in Q$, образует группу (относительно операции композиции), которую будем обозначать $\operatorname{Gr}(Q)$. В работе доказана конечная порождаемость $\operatorname{Gr}(Q)$ для большого семейства классов $Q$, удовлетворяющих определенным требованиям. В качестве примера рассмотрен класс FP функций, вычислимых за полиномиальное время на машине Тьюринга. Полученный результат обобщается на классы ஜீ $\mathscr{E}^{n}$ иерархии Гжегорчика, $n \geqslant 2$.
\end{abstract}

Кроме того, для рассматриваемых классов $Q$ определено минимальное число перестановок, порождающих группу $\operatorname{Gr}(Q)$, равное двум. Точнее, получен более сильный результат: существуют две перестановки из данной группы, композициями которых можно получить любую перестановку этой группы.

Работа выполнена при финансовой поддержке Российского фонда фундаментальных исследований, проект 06-01-00438.

\section{1. Основные определения и обозначения}

Рассматриваются всюду определенные функции на множестве $\mathbf{N}=\{0,1, \ldots\}$. Случаи, когда будут рассматриваться функции другого вида, будут оговариваться отдельно. Кроме того, по умолчанию под термином перестановка подразумевается перестановка на множестве $\mathbf{N}$.

Для одноместных функций $f, g, h$ запись $h=f \circ g$ означает, что $h(x)=f(g(x))$. Если $f$ - перестановка, то $f^{-1}$ обозначает обратную к $f$ перестановку.

Под операцией суперпозиции будем подразумевать подстановку функций в функции, перестановку и отождествление переменных, введение фиктивных переменных. Будем обозначать $[Q]$ замыкание по суперпозиции класса $Q$ функций натурального аргумента.

Для любого класса $Q$, замкнутого относительно суперпозиции и содержащего функцию $I(x)=x$, через $\operatorname{Gr}(Q)$ обозначим группу перестановок $\left(\left\{f: f, f^{-1} \in Q\right\}, \circ\right)$.

Для класса функций $Q$ будем обозначать $Q^{(1)}$ множество всех одноместных функций из $Q$.

Определение 1. Графом перестановки $f(x)$ называется ориентированный граф с множеством вершин $\mathbf{N}$ и множеством дуг $\{(x, f(x)): x \in \mathbf{N}\}$. 
Положим

$$
f^{z}= \begin{cases}f \circ \ldots \circ f, & \text { если } z>0, \\ I, & \text { если } z=0, \\ \left(f^{-1}\right)^{|z|}, & \text { если } z<0,\end{cases}
$$

где $f \circ \ldots \circ f$ содержит $z$ перестановок $f$ и $I(x)=x$ при всех $x$.

Определение 2. Перестановка $f$ называется паросочетанием на множестве $A$, если $f=f^{-1}$ и $f(x)=x$ для любого $x \notin A$. Паросочетания на множестве $\mathbf{N}$ будем называть просто паросочетаниями.

Определение 3. Характеристической функцией множества $A \subseteq \mathbf{N}$ называется функция $\chi_{A}(x)$, определяемая равенствами

$$
\chi_{A}(x)= \begin{cases}1, & \text { если } x \in A, \\ 0 & \text { в противном случае. }\end{cases}
$$

Определение 4. Перестановка $f$ называется стационарной на множестве $A$, если для любого $x \in A$ справедливо равенство $f(x)=x$.

Далее будем использовать сокращенную запись перестановок. Например, запись

$$
f: \quad g(y) \leftrightarrow h(y), \quad y \geqslant 2, \quad t(y) \rightarrow u(y) \rightarrow v(y) \rightarrow t(y)
$$

означает, что

$$
f(x)= \begin{cases}h(y), & \text { если } x=g(y) \text { при некотором } y \geqslant 2, \\ g(y), & \text { если } x=h(y) \text { при некотором } y \geqslant 2, \\ u(y), & \text { если } x=t(y) \text { при некотором натуральном } y, \\ v(y), & \text { если } x=u(y) \text { при некотором натуральном } y, \\ t(y), & \text { если } x=v(y) \text { при некотором натуральном } y, \\ x & \text { в остальных случаях. }\end{cases}
$$

Отметим, что подобная запись не всегда корректна, корректность будет доказываться в каждом отдельном случае, кроме тех случаев, когда она не вызывает сомнений.

Определение 5. Тройка $(f, g, B)$, где $f, g$ - паросочетания, $B$ - множество векторов из $\mathbf{N}^{4}$, называется правильной, если все компоненты векторов из $B$ различны (как внутри векторов, так и в различных векторах) и выполнены соотношения

$$
\begin{array}{lll}
f: & b_{1} \leftrightarrow b_{3}, \quad b_{2} \leftrightarrow b_{4}, & \left(b_{1}, b_{2}, b_{3}, b_{4}\right) \in B, \\
g: & b_{1} \leftrightarrow b_{2}, & \left(b_{1}, b_{2}, b_{3}, b_{4}\right) \in B .
\end{array}
$$

Определение 6. Правильная тройка $(f, g, B)$ называется правильной в $Q$, если $f, g \in Q$. Определение 7. Бесконечное множество $A \subseteq \mathbf{N}$ называется регулярным в классе функций $Q$, если выполняются условия

(1) $\chi_{A} \in Q$;

(2) можно так занумеровать элементы множества $A$, что функция $\mu(x)$, вычисляющая номер элемента $x$ в этой нумерации (равна нулю при $x \notin A$ ), и функция $v(x)$, вычисляющая элемент с номером $x$, принадлежат $Q$ (нумерация начинается с нуля). 
Для любого регулярного в $Q$ множества $A$ зафиксируем эти функции и обозначим их $\mu_{A}$ и $v_{A}$. Положим

$$
\begin{aligned}
x \div y & =\max (0, x-y), \\
{[x / y] } & = \begin{cases}\text { целой части от деления } x \text { на } y, & \text { если } y>0, \\
0 & \text { в противном случае, }\end{cases} \\
\operatorname{rm}(x, y) & =x \div(y \cdot[x / y]), \\
\operatorname{sg} x & = \begin{cases}1, & \text { если } x>0, \\
0 & \text { в противном случае. }\end{cases}
\end{aligned}
$$

Далее будем налагать дополнительные требования на рассматриваемые классы функций $Q$ :

(I) $Q$ содержит функции

$$
1, \quad x+y, \quad x \div y, \quad x \cdot \operatorname{sg} y, \quad[x / 2]
$$

(II) $Q$ содержит нумерационную функцию $c_{2}\left(x_{1}, x_{2}\right)$, взаимно однозначно отображающую множество $\mathbf{N}^{2}$ на $\mathbf{N}$, вместе с обратными к ней функциями $c_{2,1}(x)$ и $c_{2,2}(x)$ $\left(c_{2,1}\left(c_{2}(x, y)\right)=x, c_{2,2}\left(c_{2}(x, y)\right)=y\right.$ при любых $\left.x, y\right)$;

(III) Для любой перестановки $f \in G r(Q)$ существуют непересекающиеся регулярные в $Q$ множества $A, B$ такие, что $f(A) \cap B=\varnothing$ и $\mathbf{N} \backslash A, \mathbf{N} \backslash B$ регулярны в $Q$;

(IV) $Q$ замкнут относительно суперпозиции;

(V) $Q$ имеет конечный базис по суперпозиции.

Заметим, что требования не являются независимыми (например, IV следует из V). Тем не менее, рассматриваться будут все требования, чтобы для некоторых утверждений показать, что они справедливы при достаточно слабых ограничениях на $Q$. Кроме того, отметим, что из требований II и IV следует существование в $Q$ нумерационных функций $c_{n}\left(x_{1}, \ldots, x_{n}\right)$, взаимно однозначно отображающих $\mathbf{N}^{n}$ в $\mathbf{N}$, и обратных к ним функций $c_{n, 1}(x), \ldots, c_{n, n}(x)$ (см. [2]). Далее в тексте даны определения некоторых функций, использующие нумерационные функции, то есть зависящие от их выбора. Подразумевается, что если в каком-либо утверждении упоминается класс $Q$, удовлетворяющий требованиям II, IV, и некоторые функции из приведенных ниже, то эти функции построены на основе нумерационных функций из класса $Q$ (фиксированных для данного класса).

Для каждой функции $f(x)$ положим

$$
\begin{aligned}
\mathrm{p}_{f}: & c_{3}(x, 2 y, z) & \rightarrow c_{3}\left(f(x), 2 c_{2}(x, y), z\right), & \\
c_{3}(x, 4 y+1, z) & \rightarrow c_{3}(x, 2 y+1, z), & & \\
c_{3}(x, 4 y+3, z) & \rightarrow c_{3}(x, 2 y, z), & & x<f\left(c_{2,1}(y)\right), \\
c_{3}(x, 4 y+3, z) & \rightarrow c_{3}(x+1,2 y, z), & & x \geqslant f\left(c_{2,1}(y)\right) .
\end{aligned}
$$

Корректность этого определения будет доказана далее.

Определение 8. Паросочетание $f(x)$ называется кодом частично определенной функции $g\left(x_{1}, x_{2}\right)$, если

$$
f: c_{3}(x, y, 0) \leftrightarrow c_{3}(x, y, g(x, y)+2),
$$

где $g(x, y)$ определено. 
Обозначим рх код функции $g\left(x_{1}, x_{2}\right)=x_{1}$. Положим

$$
\begin{aligned}
& \text { del: } \quad c_{3}(x, 2 y, 0) \leftrightarrow c_{3}(x, 2 y, 1), \\
& \mathrm{s}_{i j}: \quad 4 x+i \leftrightarrow 4 x+j, \quad 0 \leqslant i<j<3, \\
& \text { move: } \quad c_{3}(x, 2 y, 0) \rightarrow c_{3}(x, 2 y+2,0) \text {, } \\
& c_{3}(x, 1,0) \rightarrow c_{3}(x, 0,0), \\
& c_{3}(x, 2 y+3,0) \rightarrow c_{3}(x, 2 y+1,0), \\
& \text { place: } \quad c_{3}(x, 0,0) \rightarrow 2 x \text {, } \\
& c_{3}(x, y+1,0) \rightarrow 4 c_{2}(x, y)+1 \text {, } \\
& c_{3}(x, y, z+1) \rightarrow 4 c_{3}(x, y, z)+3 \text {, } \\
& \operatorname{swap}_{1} \text { : } \quad c_{3}(x, 2 y, z) \rightarrow c_{3}(x+2,2 y, z), \quad z \geqslant 2 \text {, } \\
& c_{3}(x, 2 y, 0) \rightarrow c_{3}(x, 2 y, 0), \\
& c_{3}(x+2,2 y+1, z) \rightarrow c_{3}(x, 2 y+1, z), \quad z \geqslant 2, \\
& c_{3}(x, 2 y+1, z) \rightarrow c_{3}(x, 2 y, z), x \in\{0,1\}, \quad z \geqslant 2, \\
& \operatorname{swap}_{2} \text { : } \quad c_{3}(x, 2 y, z) \rightarrow c_{3}(z, 2 y, x+2), \quad z \geqslant 2 \text {, } \\
& c_{3}(x, 2 y, 0) \rightarrow c_{3}(x, 2 y, 0), \\
& c_{3}(x+2,2 y+1, z) \rightarrow c_{3}(x, 2 y+1, z), \quad z \geqslant 2, \\
& c_{3}(x, 2 y+1, z) \rightarrow c_{3}(x, 2 y, z), x \in\{0,1\}, \quad z \geqslant 2 .
\end{aligned}
$$

\section{2. Конечная порождаемость группы $G r(Q)$}

Предложение 1. Определение $\mathrm{p}_{f}$ корректно, $и \mathrm{p}_{f}-$ перестановка для любой функции $f(x)$. Кроме того, если $Q$ удовлетворяет требованиям I, II, IV $u f \in Q$, mо $\mathrm{p}_{f} \in G r(Q)$.

Доказательство. Докажем, что определение (3) корректно и $\mathrm{p}_{f}-$ перестановка. Действительно, нетрудно заметить, что $i$-е правило в (3), $1 \leqslant i \leqslant 4$, взаимно однозначно отображает множество $A_{i}$ на $B_{i}$, где

$$
\begin{aligned}
& A_{1}=\left\{c_{3}(u, v, w): \operatorname{rm}(v, 2)=0\right\}, \\
& A_{2}=\left\{c_{3}(u, v, w): \operatorname{rm}(v, 4)=1\right\}, \\
& A_{3}=\left\{c_{3}(u, v, w): \operatorname{rm}(v, 4)=3, u<f\left(c_{2,1}([v / 4])\right)\right\}, \\
& A_{4}=\left\{c_{3}(u, v, w): \operatorname{rm}(v, 4)=3, u \geqslant f\left(c_{2,1}([v / 4])\right)\right\}, \\
& B_{1}=\left\{c_{3}(u, v, w): \operatorname{rm}(v, 2)=0, u=f\left(c_{2,1}([v / 2])\right)\right\}, \\
& B_{2}=\left\{c_{3}(u, v, w): \operatorname{rm}(v, 2)=1\right\}, \\
& B_{3}=\left\{c_{3}(u, v, w): \operatorname{rm}(v, 2)=0, u<f\left(c_{2,1}([v / 2])\right)\right\}, \\
& B_{4}=\left\{c_{3}(u, v, w): \operatorname{rm}(v, 2)=0, u>f\left(c_{2,1}([v / 2])\right)\right\} .
\end{aligned}
$$

Ясно, что $\left\{A_{1}, A_{2}, A_{3}, A_{4}\right\},\left\{B_{1}, B_{2}, B_{3}, B_{4}\right\}$ - разбиения множества $\mathbf{N}$. Из этого следует корректность определения и то, что $\mathrm{p}_{f}-$ перестановка.

Доказательство того, что $\mathrm{p}_{f}, \mathrm{p}_{f}^{-1} \in Q$, следует непосредственно из (3) (см. [4]). Предложение доказано.

Доказательства предложений 2 и 3 аналогичны доказательству предложения 1. 


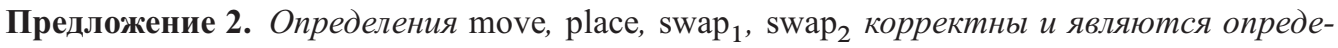
лениями перестановок. Кроме того, если класс $Q$ удовлетворяет требованиям I, II, IV, то эти перестановки принадлежат $\operatorname{Gr}(Q)$.

Предложение 3. Если класс $Q$ удовлетворяет требованиям I, II, IV, mо del $=\operatorname{del}^{-1} \in Q$, $\mathrm{s}_{i j}=\mathrm{s}_{i j}^{-1} \in Q, 0 \leqslant i<j \leqslant 3, \mathrm{px}=\mathrm{px}^{-1} \in Q$.

Предложение 4. Пусть класс $Q$ удовлетворяет требованиям I, IV. Тогда любое регулярное в $Q$ множество $А$ можно разбить на два регулярных в $Q$ множества $A_{1} u A_{2}$.

Доказательство. Для $i=1,2$ положим

$$
\begin{aligned}
A_{i} & =\left\{x: x \in A, \mu_{A}(x) \equiv i-1 \quad(\bmod 2)\right\}, \\
\mu_{A_{i}}(x) & = \begin{cases}\left(\mu_{A}(x)-i+1\right) / 2, & \text { если } x \in A_{i}, \\
0 & \text { в противном случае, }\end{cases} \\
v_{A_{i}}(x) & =v_{A}(2 x+i-1) .
\end{aligned}
$$

Из I и IV следует, что $\chi_{A_{i}}, \mu_{A_{i}}, v_{A_{i}} \in Q$ при $i=1,2$. Ясно, что функции $\mu_{A_{i}}, v_{A_{i}}$ подходят под определение регулярности для $A_{i}$. Кроме того, очевидно, $A_{1}, A_{2}$ бесконечны и образуют разбиение $A$. Предложение доказано.

Предложение 5. Пусть класс $Q$ удовлетворяет требованиям I, IV. Тогда для любых непересекающихся регулярных в $Q$ множеств $A$ и $B$ множество $A \cup B$ регулярно в $Q$.

Доказательство. Положим

$$
\begin{aligned}
& \mu(x)= \begin{cases}2 \mu_{A}(x), & \text { если } x \in A, \\
2 \mu_{B}(x)+1, & \text { если } x \in B, \\
0 & \text { в противном случае, }\end{cases} \\
& v(x)= \begin{cases}v_{A}(x / 2), & \text { если } x \equiv 0 \\
v_{B}((x-1) / 2), & \text { если } x \equiv 1 \quad(\bmod 2)),\end{cases}
\end{aligned}
$$

Из требований I и IV следует, что $\mu, v \in Q$. Ясно, что функции $\mu, v$ подходят под определение регулярности для $A \cup B, \chi_{A \cup B}=\chi_{A}+\chi_{B} \in Q$. Предложение доказано.

Предложение 6. Пусть класс $Q$ удовлетворяет требованиям I-IV. Тогда для любой перестановки $f \in G r(Q)$ существуют перестановки $f_{1}, f_{2} \in G r(Q)$ и регулярные в $Q$ множества $A_{1}, A_{2}$ такие, что $\mathbf{N} \backslash A_{1}, \mathbf{N} \backslash A_{2}$ регулярны в $Q, f=f_{1} \circ f_{2}, f_{i}$ стационарна на множестве $A_{i}, i=1,2$.

Доказательство. Пусть $A, B$ - множества из требования III для $Q$ и $f$. Разобьем $B$ на два регулярных в $Q$ множества $B_{1}, B_{2}$ (это возможно в силу предложения 4 ).

Положим

$$
\begin{aligned}
& f_{1}: \quad x \rightarrow f(x), \quad x \in A ; \\
& x \rightarrow v_{B_{1}}\left(c_{2}(x, 0)\right), \quad x \notin A, \quad f^{-1}(x) \in A ; \\
& v_{B_{1}}\left(c_{2}(x, 0)\right) \rightarrow x, \quad x \in A, \quad f^{-1}(x) \notin A ; \\
& v_{B_{1}}\left(c_{2}(x, y)\right) \rightarrow v_{B_{1}}\left(c_{2}(x, y+1)\right), \quad x \notin A, \quad f^{-1}(x) \in A ; \\
& v_{B_{1}}\left(c_{2}(x, y+1)\right) \rightarrow v_{B_{1}}\left(c_{2}(x, y)\right), \quad x \in A, \quad f^{-1}(x) \notin A \text {. }
\end{aligned}
$$


Легко показать, что множество дуг графа перестановки $f_{1}$ получается из множества $\{(x, f(x)): x \in A\}$ добавлением для каждой вершины $x$, являющейся стоком этого множества (вершины, в которую входит дуга, но из которой не выходит) бесконечной цепочки $x \rightarrow v_{B_{1}}\left(c_{2}(x, 0)\right) \rightarrow v_{B_{1}}\left(c_{2}(x, 1)\right) \rightarrow \ldots$, для каждой вершины $x$, являющейся истоком (вершины, из которой выходит дуга, но не входит) бесконечной цепочки $\ldots \rightarrow v_{B_{1}}\left(c_{2}(x, 1)\right) \rightarrow v_{B_{1}}\left(c_{2}(x, 0)\right) \rightarrow x$ и добавлением петель $(x, x)$ для каждой вершины $x$, из которой не выходит дуга и в которую не входит дуга. После этого построения получается граф, у которого для каждой вершины существует ровно одна выходящая и ровно одна входящая дуга. Из этого следует, что определение $f_{1}$ корректно и $f_{1}-$ перестановка.

Из определения $f_{1}$ следует, что $f_{1}$ стационарна на множестве $\mathbf{N} \backslash\left(A \cup f(A) \cup B_{1}\right)$. Из требования III для $Q$ следует, что $(A \cup f(A)) \cap B=\varnothing$. Поэтому $B_{2} \subseteq \mathbf{N} \backslash\left(A \cup f(A) \cup B_{1}\right)$. Отсюда вытекает, что $f_{1}$ стационарна на $B_{2}$.

Пусть $f_{2}=f_{1}^{-1} \circ f$. Из того, что $f_{1}$ совпадает с $f$ на $A$ (см. определение $f_{1}$ ) следует, что $f_{2}$ стационарна на $A$.

По аналогии с предложением 1 легко показать, что $f_{1}, f_{1}^{-1} \in Q$. Из этого следует, что $f_{2} \in Q$ и $f_{2}^{-1}=f^{-1} \circ f_{1} \in Q$. Положим $A_{1}=B_{2}, A_{2}=A$. Заметим, что регулярность $\mathbf{N} \backslash A_{2}$ в $Q$ следует из выбора множества $A$, регулярность $\mathbf{N} \backslash A_{1}=(\mathbf{N} \backslash B) \cup B_{1}-$ из выбора множества $B$, регулярности $B_{1}$ и предложения 5. Предложение доказано.

Предложение 7. Пусть класс $Q$ удовлетворяет требованиям I, II, IV. Тогда, если перестановка $f \in G r(Q)$ стационарна на некотором регулярном в $Q$ множестве $A$ таком, что $\mathbf{N} \backslash A$ тоже регулярно в $Q$, то существуют правильные в $Q$ тройки $\left(f_{1}, g_{1}, B_{1}\right), \ldots,\left(f_{k}, g_{k}, B_{k}\right)$ такие, что $f=f_{1} \circ \ldots \circ f_{k}$.

Доказательство. Применив два раза предложение 4, разобьем множество $A$ на регулярные в $Q$ множества $A_{1}, A_{2}, A_{3}$.

Введем вспомогательные функции $c^{\prime}$ и $c^{\prime \prime}$, отображающие $\mathbf{N} \times \mathbf{Z}$, где $\mathbf{Z}-$ множество целых чисел, в $\mathbf{N}$,

$$
\begin{gathered}
c^{\prime}(x, z)= \begin{cases}v_{\mathbf{N} \backslash A}(x), & \text { если } z=0, \\
v_{A_{1}}(x), & \text { если } z=1, \\
v_{A_{3}}\left(c_{2}(x, z-2)\right), & \text { если } z>1, \\
v_{A_{2}}\left(c_{2}(x,-z-1)\right), & \text { если } z<0,\end{cases} \\
c^{\prime \prime}(x, z)= \begin{cases}c^{\prime}(x, z), & \text { если } z \leqslant 0, \\
c^{\prime}\left(\mu_{\mathbf{N} \backslash A} \circ f \circ v_{\mathbf{N} \backslash A}(x), z\right), & \text { если } z>0 .\end{cases}
\end{gathered}
$$

Очевидно, что отображение $c^{\prime}$ взаимно однозначно. Кроме того, ясно, что $f$ взаимно однозначно отображает $\mathbf{N} \backslash A$ на $\mathbf{N} \backslash A$ (потому что $f$ стационарна на $A$ ). Следовательно, $\mu_{\mathbf{N} \backslash A} \circ f \circ v_{\mathbf{N} \backslash A}-$ перестановка $\left(v_{\mathbf{N} \backslash A}\right.$ взаимно однозначно отображает $\mathbf{N}$ на $\mathbf{N} \backslash A, f-$ $\mathbf{N} \backslash A$ на $\mathbf{N} \backslash A$, a $\mu_{\mathbf{N} \backslash A}-\mathbf{N} \backslash A$ на $\left.\mathbf{N}\right)$. Из этого следует, что $c^{\prime \prime}$ тоже взаимно однозначно.

Положим

$$
\begin{array}{lll}
r_{1}: & c^{\prime \prime}(x, z) \leftrightarrow c^{\prime \prime}(x, 1-z), & z \in \mathbf{Z}, \\
r_{2}: & c^{\prime \prime}(x, z) \leftrightarrow c^{\prime \prime}(x,-z), & z \in \mathbf{Z} .
\end{array}
$$

Перестановки $h_{1}, h_{2}$ определим такими же формулами с заменой $c^{\prime \prime}$ на $c^{\prime}$. Отметим, что $r_{1}$, $r_{2}, h_{1}, h_{2}$ - паросочетания, принадлежащие $Q$ (доказывается аналогично предложению 1 ). 
Положим $r=r_{1} \circ r_{2}, h=h_{1} \circ h_{2}$. Проверкой убеждаемся, что

$$
\begin{aligned}
r: \quad c^{\prime \prime}(x, z) & \rightarrow c^{\prime \prime}(x, z+1), \\
h: \quad c^{\prime}(x, z) & \rightarrow c^{\prime}(x, z+1) .
\end{aligned}
$$

Из (11), (12) и из того, что $\mu_{\mathbf{N} \backslash A} \circ f \circ v_{\mathbf{N} \backslash A}-$ перестановка, вытекает, что множество дуг графа перестановки $r$ представляет собой объединение всех бесконечных цепочек вида $\ldots \rightarrow c^{\prime}(x,-1) \rightarrow c^{\prime}(x, 0), c^{\prime}(x, 1) \rightarrow c^{\prime}(x, 2) \rightarrow \ldots$, где $x \in \mathbf{N}$, и множества $\left\{\left(c^{\prime}(x, 0), c^{\prime}\left(\mu_{\mathbf{N} \backslash A} \circ f \circ v_{\mathbf{N} \backslash A}(x), 1\right)\right), x \in \mathbf{N}\right\}$. С другой стороны, из (13) следует, что множество всех дуг графа перестановки $h$ представляет собой объединение непересекающихся бесконечных цепочек вида $\ldots \rightarrow c^{\prime}(x,-1) \rightarrow c^{\prime}(x, 0) \rightarrow c^{\prime}(x, 1) \rightarrow \ldots$, где $x \in \mathbf{N}$. Из этого вытекает, что $r$ и $h$ совпадают на множестве $\left\{c^{\prime}(x, z): z \in \mathbf{Z}, z \neq 0\right\}=A$ (см. (10)). Отсюда можно сделать вывод, что $h^{-1} \circ g$ стационарна на $A$.

Далее, при любом $x \in \mathbf{N}$ справедливы равенства

$$
r\left(c^{\prime}(x, 0)\right)=r\left(c^{\prime \prime}(x, 0)\right)=c^{\prime \prime}(x, 1)=c^{\prime}\left(\mu_{\mathbf{N} \backslash A} \circ f \circ v_{\mathbf{N} \backslash A}(x), 1\right)
$$

(равенства следуют из (11), (12), (11) соответственно). Далее,

$$
\begin{aligned}
h^{-1} \circ r \circ v_{\mathbf{N} \backslash A}(x) & =h^{-1} \circ r\left(c^{\prime}(x, 0)\right)=h^{-1}\left(c^{\prime}\left(\mu_{\mathbf{N} \backslash A} \circ f \circ v_{\mathbf{N} \backslash A}(x), 1\right)\right) \\
& =c^{\prime}\left(\mu_{\mathbf{N} \backslash A} \circ f \circ v_{\mathbf{N} \backslash A}(x), 0\right)=v_{\mathbf{N} \backslash A} \circ \mu_{\mathbf{N} \backslash A} \circ f \circ v_{\mathbf{N} \backslash A}(x) \\
& =f \circ v_{\mathbf{N} \backslash A}(x)
\end{aligned}
$$

(равенства от первого до четвертого следуют из (10), (14), (13), (10) соответственно). Таким образом, $h^{-1}$ or и $f$ совпадают на $\mathbf{N} \backslash A$. С учетом того, что $f$ и $h^{-1}$ or стационарны на $A$, получаем, что $f=h^{-1} \circ r=h_{2} \circ h_{1} \circ r_{1} \circ r_{2}$. Пусть

$$
\begin{gathered}
s_{1}: \quad c^{\prime}(x,-2 y) \leftrightarrow c^{\prime}(x,-2 y-1), \quad y \geqslant 0, \\
s_{2}: \quad c^{\prime}(x,-2 y-1) \leftrightarrow c^{\prime}(x,-2 y-2), \quad y \geqslant 0, \\
M_{1}=\left\{\left(c^{\prime}(x,-2 y), c^{\prime}(x,-2 y-1), c^{\prime}(x, 2 y+1), c^{\prime}(x, 2 y+2)\right), y \geqslant 0\right\}, \\
M_{2}=\left\{\left(c^{\prime}(x,-2 y-1), c^{\prime}(x,-2 y-2), c^{\prime}(x, 2 y+1), c^{\prime}(x, 2 y+2)\right), y \geqslant 0\right\} .
\end{gathered}
$$

Ясно, что $s_{1}, s_{2}$ - паросочетания в $Q$. Поэтому нетрудно заметить, что $\left(r_{1}, s_{1}, M_{1}\right)$, $\left(r_{2}, s_{2}, M_{2}\right),\left(h_{1}, s_{1}, M_{1}\right),\left(h_{2}, s_{2}, M_{2}\right)$ - правильные в $Q$ тройки. Предложение доказано.

Теорема 1. Пусть класс $Q$ удовлетворяет требованиям I-IV. Тогда для любой перестановки $f \in \operatorname{Gr}(Q)$ найдутся правильные в $Q$ тройки $\left(f_{1}, g_{1}, B_{1}\right), \ldots,\left(f_{k}, g_{k}, B_{k}\right)$ такие, что $f=f_{1} \circ \ldots \circ f_{k}$.

Доказательство. Используя предложение 6, представим $f$ в виде композиции перестановок, лежащих в $Q$ вместе с обратными им перестановками, стационарных на некоторых множествах, регулярных вместе со своими дополнениями. Затем с использованием предложения 7 представим каждую из этих перестановок в виде композиции разбиваемых в $Q$ паросочетаний. Теорема доказана.

Предложение 8. Пусть перестановка $f$ корректно определяется формулой

$$
f: g_{1}\left(\tilde{x_{1}}\right) \rightarrow h_{1}\left(\tilde{x_{1}}\right), \rho_{1}\left(\tilde{x_{1}}\right) \text { истинно, } \ldots, g_{n}\left(\tilde{x_{n}}\right) \rightarrow h_{n}\left(\tilde{x_{n}}\right), \rho_{n}\left(\tilde{x_{n}}\right) \text { истинно }
$$

где $g_{i}, h_{i}$ - некоторые функции, $\rho_{i}-$ некоторые предикаты, $1 \leqslant i \leqslant n$. Кроме того, пусть р-некоторая перестановка. Тогда

$$
\begin{aligned}
p \circ f \circ p^{-1}: \quad p\left(g_{1}\left(\tilde{x_{1}}\right)\right) \rightarrow p\left(h_{1}\left(\tilde{x_{1}}\right)\right) \rho_{1}\left(\tilde{x_{1}}\right) \text { истинно, } \ldots, \\
p\left(g_{n}\left(\tilde{x_{n}}\right)\right) \rightarrow p\left(h_{n}\left(\tilde{x_{n}}\right)\right), \rho_{n}\left(\tilde{x_{n}}\right) \text { истинно. }
\end{aligned}
$$


Справедливость предложения подтверждается прямой проверкой.

Предложение 9. Пусть $g(x, y)$ - частично определенная функиия, $f$ - перестановка, являющаяся ее кодом. Кроме того, пусть $\left(h_{1}(x, y), h_{2}(x, y)\right)$ - перестановка на множестве $\mathbf{N}^{2}, p-$ перестановка, определяемая формулой

$$
p: \quad c_{3}(x, y, z) \rightarrow c_{3}\left(h_{1}(x, y), h_{2}(x, y), z\right) .
$$

Тогда $p^{-1} \circ f \circ p$ является кодом функичи $g\left(h_{1}(x, y), h_{2}(x, y)\right)$.

Доказательство. Пусть $\left(h_{1}^{\prime}, h_{2}^{\prime}\right)$ - обратная перестановка к $\left(h_{1}, h_{2}\right)$, то есть

$$
h_{1}^{\prime}\left(h_{1}(x, y), h_{2}(x, y)\right)=x, \quad h_{2}^{\prime}\left(h_{1}(x, y), h_{2}(x, y)\right)=y
$$

для любых $x, y$. Ясно, что $p^{-1}: c_{3}(x, y, z) \rightarrow c_{3}\left(h_{1}^{\prime}(x, y), h_{2}^{\prime}(x, y), z\right)$. Используя предложение 8 , получаем, что

$$
p^{-1} \circ f \circ p: \quad c_{3}\left(h_{1}^{\prime}(x, y), h_{2}^{\prime}(x, y), 0\right) \leftrightarrow c_{3}\left(h_{1}^{\prime}(x, y), h_{2}^{\prime}(x, y), g(x, y)+2\right),
$$

где $g(x, y)$ определено. С учетом того, что $\left(h_{1}^{\prime}, h_{2}^{\prime}\right)$ - перестановка, можно сделать замену переменных $z=h_{1}^{\prime}(x, y), t=h_{2}^{\prime}(x, y)$ и получить, что

$$
p^{-1} \circ f \circ p: \quad c_{3}(z, t, 0) \leftrightarrow c_{3}\left(z, t, g\left(h_{1}(z, t), h_{2}(z, t)\right)+2\right),
$$

где $g\left(h_{1}(z, t), h_{2}(z, t)\right)$ определено. Предложение доказано.

Предложение 10. Пусть $f(x), g(x)$ - некоторые функции, $r\left(x_{1}, x_{2}\right)-$ функция, для которой при любых $x, y$ справедливо равенство $r(x, 2 y)=f(x), s-$ перестановка, являющался кодом $r$. Тогда $\mathrm{p}_{g}^{-1}$ оs о $\mathrm{p}_{g}$ - перестановка, являющаяся кодом такой функиии $q\left(x_{1}, x_{2}\right)$, что $q(x, 2 y)=f(g(x))$.

Доказательство. Из определения перестановки $\mathrm{p}_{g}$ (см. (3)) следует, что

$$
\mathrm{p}_{g}: \quad c_{3}(x, y, z) \rightarrow c_{3}\left(h_{1}(x, y), h_{2}(x, y), z\right),
$$

где $\left(h_{1}, h_{2}\right)$ - перестановка на $\mathbf{N}^{2}$, причем при любых $x, y$ имеют место равенства

$$
h_{1}(x, 2 y)=g(x), \quad h_{2}(x, 2 y)=2 c_{2}(x, y)
$$

(равенства следуют из первого правила в (3)). Используя предложение 9, получаем, что

$$
q(x, 2 y)=r\left(h_{1}(x, 2 y), h_{2}(x, 2 y)\right)=r\left(g(x), 2 c_{2}(x, y)\right)=f(g(x)) .
$$

Предложение доказано.

Предложение 11. Пусть $f_{1}(x), \ldots, f_{n}(x)-$ функциии, $f=f_{1} \circ \ldots \circ f_{n} u$

$$
p=\mathrm{p}_{f_{n}}^{-1} \circ \ldots \circ \mathrm{p}_{f_{1}}^{-1} \circ \mathrm{px} \circ \mathrm{p}_{f_{1}} \circ \ldots \circ \mathrm{p}_{f_{n}} .
$$

Тогда р есть код функиии $g(x, y)$, причем $g(x, 2 y)=f(x)$ при любых $x, y$.

Доказательство. По определению перестановка рх есть код функции $p^{\prime}(x, y)=x$, поэтому при любых $x$ и $y$ имеет место равенство $p^{\prime}(x, 2 y)=x$. Таким образом, применив $n$ раз предложение 10 , получим доказываемое утверждение. 
Предложение 12. Пусть $f_{1}, f_{2}$ - паросочетания, $A \subseteq \mathbf{N}$. Кроме того, пусть выполнень следующие условия:

(1) $A, f_{1}(A)$ и $f_{2}(A)$ попарно не пересекаются;

(2) $f_{1}$ стационарна на $f_{2}(A)$;

(3) $f_{2}$ стационарна на $\mathbf{N} \backslash\left(A \cup f_{2}(A)\right)$.

Тогда $\left(f_{1} \circ f_{2}\right)^{4} \circ f_{2}: x \leftrightarrow f_{1}(x), x \in A$.

Доказательство. Ясно, что множество всех дуг (исключая петли) графа перестановки $f_{1}$ состоит из пар дуг вида $x \leftrightarrow f_{1}(x), x \in A$, и еще некоторых пар дуг вида $x \leftrightarrow y$, где $x, y \notin A \cup f_{1}(A) \cup f_{2}(A)$. Аналогичное множество для $f_{2}$ состоит только из пар дуг $x \leftrightarrow f_{2}(x), x \in A$. Из этого с учетом условия 1 следует, что граф перестановки $f_{1} \circ f_{2}$ состоит из непересекающихся циклов длины 3 вида $x \rightarrow f_{2}(x) \rightarrow f_{1}(x) \rightarrow x, x \in A$, циклов длины 2 вида $x \leftrightarrow f_{1}(x), x, f_{1}(x) \notin A \cup f_{1}(A) \cup f_{2}(A)$ и петель. После возведения в 4-ю степень циклы длины 3 и петли останутся на месте, циклы длины 2 преобразуются в пары петель. После умножения результата на $f_{2}$ циклы длины 3 преобразуются в циклы длины 2 вида $x \leftrightarrow f_{1}(x), x \in A$, и петли $f_{2}(x) \rightarrow f_{2}(x), x \in A$, все остальное останется на месте. Предложение доказано.

Предложение 13. Если $f$ - перестановка, являющаяся кодом всюду определенной функциии $g\left(x_{1}, x_{2}\right)$, то $(f \circ \mathrm{del})^{4} \circ \mathrm{del}$ есть код частично определенной функичи $g^{\prime}\left(x_{1}, x_{2}\right)$, где

$$
g^{\prime}\left(x_{1}, x_{2}\right)= \begin{cases}g\left(x_{1}, x_{2}\right), & \text { если } x_{2} \text { четно, } \\ \text { не определено } & \text { в противном случае. }\end{cases}
$$

Доказательство. Пусть $A=\left\{c_{3}(x, 2 y, 0), x, y \in \mathbf{N}\right\}$. Из (4) и (5) следует, что

$$
f(A) \subseteq\left\{c_{3}(x, 2 y, z): z \geqslant 2\right\}, \quad \operatorname{del}(A)=\left\{c_{3}(x, 2 y, 1): x, y \in \mathbf{N}\right\} .
$$

Таким образом, $A, f(A), \operatorname{del}(A)$ попарно не пересекаются. Кроме того, легко проверить, что $f$ стационарна на $\left\{c_{3}(x, 2 y, 1): x, y \in \mathbf{N}\right\}$, a del - на $\left\{c_{3}(x, 2 y, z): z \geqslant 2\right\}$. Таким образом, выполнены все условия предложения 12 для множества $A$ и функций $f$, del. Из этого следует, что $(f \circ \mathrm{del})^{4} \circ \mathrm{del}: x \leftrightarrow f(x), x \in A$. Это можно переписать в виде $(f \circ \mathrm{del})^{4} \circ \mathrm{del}: c_{3}(x, 2 y, 0) \leftrightarrow c_{3}(x, 2 y, g(x, 2 y)+2)$. Предложение доказано.

Предложение 14. Пусть класс $Q$ удовлетворяет требованиям I, II $u$ IV, $u$ пусть $Q^{(1)}=\left[\left\{q_{1}, \ldots, q_{n}\right\}\right]$. Тогда любое паросочетание на множестве всех четных чисел $f \in Q$ представимо в виде композиции перестановок $\mathrm{p}_{q_{1}}, \ldots, \mathrm{p}_{q_{n}}, \mathrm{px}, \operatorname{swap}_{1}, \operatorname{swap}_{2}$, move, place, del и им обратных.

Доказательство. Пусть

$$
f^{\prime}(x)=f(2 x) / 2
$$

Ясно, что $f^{\prime}(x)$ - паросочетание, принадлежащее Q. Пусть $f^{\prime}=r_{1} \circ \ldots \circ r_{k}$, где $r_{1}, \ldots, r_{k} \in\left\{q_{1}, \ldots, q_{n}\right\}$. Пусть $\psi=\mathrm{p}_{r_{k}}^{-1} \circ \ldots \circ \mathrm{p}_{r_{1}}^{-1} \circ \mathrm{px} \circ \mathrm{p}_{r_{1}} \circ \ldots \circ \mathrm{p}_{r_{k}}$. Из предложения 11 следует, что $\psi$ является кодом некоторой функции $g(x, y)$, причем для любых $x$ и $y$

$$
g(x, 2 y)=f^{\prime}(x) .
$$


Положим $\psi_{1}=(\psi \circ \mathrm{del})^{4} \circ \mathrm{del}$. Из предложения 13 и (16) следует, что $\psi_{1}-$ код частично определенной функции $g^{\prime}(x, y)$, задаваемой равенством

$$
g^{\prime}(x, y)= \begin{cases}f^{\prime}(x), & \text { если } y \text { четно, } \\ \text { не определено } & \text { в противном случае. }\end{cases}
$$

Из этого следует, что

$$
\psi_{1}: \quad c_{3}(x, 2 y, 0) \leftrightarrow c_{3}\left(x, 2 y, f^{\prime}(x)+2\right) .
$$

Пусть $\psi_{2}=\operatorname{swap}_{1} \circ \psi_{1} \circ \operatorname{swap}_{1}^{-1}, \psi_{3}=\operatorname{swap}_{2} \circ \psi_{1} \circ \operatorname{swap}_{2}^{-1}$. Из первых двух правил в формулах (8) и (9), (17) и предложения 8 следует, что

$$
\begin{array}{ll}
\psi_{2}: & c_{3}(x, 2 y, 0) \leftrightarrow c_{3}\left(x+2,2 y, f^{\prime}(x)+2\right), \\
\psi_{3}: & c_{3}(x, 2 y, 0) \leftrightarrow c_{3}\left(f^{\prime}(x)+2,2 y, x+2\right) .
\end{array}
$$

С учетом того, что $f^{\prime}-$ паросочетание, сделаем замену $u=f^{\prime}(x)$ и перепишем (19) в виде

$$
\psi_{3}: \quad c_{3}\left(f^{\prime}(u), 2 y, 0\right) \leftrightarrow c_{3}\left(u+2,2 y, f^{\prime}(u)+2\right)
$$

Пусть $\psi_{4}=\psi_{3} \circ \psi_{2}$. Из (18), (20) и того, что $f^{\prime}$ - паросочетание, следует, что

$$
\text { 将: } \begin{aligned}
c_{3}(x, 2 y, 0) & \leftrightarrow c_{3}\left(f^{\prime}(x), 2 y, 0\right), \\
c_{3}\left(x+2,2 y, f^{\prime}(x)+2\right) & \leftrightarrow c_{3}\left(f^{\prime}(x)+2,2 y, x+2\right) .
\end{aligned}
$$

Пусть $\psi_{5}=$ move $о \psi_{4} \circ$ move $^{-1}$. Из (6), (21) и предложения 8 следует, что

$$
\begin{aligned}
& \psi_{5}: \quad c_{3}(x, 2 y, 0) \leftrightarrow c_{3}\left(f^{\prime}(x), 2 y, 0\right), \quad y>0 \text {, } \\
& c_{3}\left(x+2,2 y, f^{\prime}(x)+2\right) \leftrightarrow c_{3}\left(f^{\prime}(x)+2,2 y, x+2\right) .
\end{aligned}
$$

Положим

$$
\psi_{6}: \quad c_{3}(x, 0,0) \leftrightarrow c_{3}\left(f^{\prime}(x), 0,0\right) .
$$

Из (21), (22) и равенства $\psi_{5}=\psi_{5}^{-1}$ следует, что $\psi_{6}=\psi_{5} \circ \psi_{4}$. Из первого правила в (7), (23) и предложения 8 следует, что place $\circ \psi_{6} \circ$ place $^{-1}: 2 x \leftrightarrow 2 f^{\prime}(x)$. Из этого и (15) вытекает, что $f=$ place $\circ \psi_{6} \circ$ place $^{-1}$. Предложение доказано.

Теорема 2. Пусть класс $Q$ удовлетворяет требованиям I-IV $u Q^{(1)}=\left[\left\{q_{1}, \ldots, q_{n}\right\}\right]$. Тогда любая перестановка $f \in G r(Q)$ представима в виде композиции перестановок $\mathrm{p}_{q_{1}}, \ldots, \mathrm{p}_{q_{n}}, \mathrm{px}, \mathrm{swap}_{1}$, $\mathrm{swap}_{2}$, move, place, del, $\mathrm{s}_{01}, \mathrm{~s}_{02}, \mathrm{~s}_{03}, \mathrm{~s}_{12}, \mathrm{~s}_{13}, \mathrm{~s}_{23}$ и им обратных.

Доказательство. По теореме 1 можно считать, что $f$ - паросочетание. Пусть

$$
f_{i j}: \quad x \leftrightarrow f(x), \quad x \equiv i \quad(\bmod 4), \quad f(x) \equiv j \quad(\bmod 4), \quad 0 \leqslant i \leqslant j \leqslant 3 .
$$

Ясно, что $f_{i j}$ - паросочетания, $f=f_{00} \circ f_{01} \circ \ldots \circ f_{33}$.

Очевидно, что для любых $i, j, 0 \leqslant i \leqslant j \leqslant 3$, существует перестановка $q_{i j}$, представимая в виде композиции перестановок $\mathrm{s}_{01}, \mathrm{~s}_{02}, \mathrm{~s}_{03}, \mathrm{~s}_{12}, \mathrm{~s}_{13}, \mathrm{~s}_{23}$, переводящая множество 
$\{x: \operatorname{rm}(x, 4) \in\{i, j\}\}$ в некоторое подмножество множества всех четных чисел (заметим, что $q_{i j}^{-1}$ тоже представима в виде композиции этих перестановок). Пусть

$$
f_{i j}^{\prime}=q_{i j} \circ f_{i j} \circ q_{i j}^{-1}, \quad 0 \leqslant i \leqslant j \leqslant 3 .
$$

Из предложения 8 и того, что $f_{i j}$ - паросочетание на множестве $\{x: \operatorname{rm}(x, 4) \in\{i, j\}\}$ следует, что $f_{i j}^{\prime}$ - паросочетание на множестве всех четных чисел. Следовательно, при любых $i, j, 0 \leqslant i \leqslant j \leqslant 3, f_{i j}^{\prime}$ представимо в виде композиции перестановок из условия теоремы (см. предложение 14). Из того, что

$$
f_{i j}=q_{i j}^{-1} \circ f_{i j}^{\prime} \circ q_{i j}, \quad 0 \leqslant i \leqslant j \leqslant 3,
$$

вытекает утверждение теоремы.

Следствие 1. Если класс $Q$ удовлетворяет требованиям I-III, V, то группа $G r(Q)$ является конечно порождаемой.

Доказательство. Действительно, из [3] известно, что из требований I, II, V вытекает существование конечного базиса по суперпозиции в $Q^{(1)}$. Остается применить теорему 2.

\section{3. Порождаемость $\operatorname{Gr}(Q)$ двумя перестановками}

Предложение 15. Пусть класс $Q$ удовлетворяет требованиям I, IV. Кроме того, пусть $A, B, C$ - непересекаюшиеся множества, B регулярно в $Q$. Тогда любая перестановка $f \in Q$, являюшаяся паросочетанием на множестве $A \cup B \cup C$, представима в виде композиции перестановок из $Q$, каждая из которых - паросочетание на множестве $A \cup B$ или $B \cup C$.

Доказательство. Пусть

$$
\begin{array}{ll}
f_{1}: & x \leftrightarrow f(x), \quad x, f(x) \in A \cup B, \\
f_{2}: & x \leftrightarrow f(x), \quad x \in C, \quad f(x) \in B \cup C, \\
f_{3}: & x \leftrightarrow f(x), \quad x \in A, \quad f(x) \in C .
\end{array}
$$

Ясно, что $f=f_{1} \circ f_{2} \circ f_{3}$. Положим

$$
\begin{aligned}
& g_{1}: \quad x \leftrightarrow v_{B}(x), \quad x \in A, \quad f(x) \in C, \\
& g_{2}: \quad v_{B}(x) \leftrightarrow f(x), \quad x \in A, \quad f(x) \in C .
\end{aligned}
$$

Отметим, что $v_{B}(x)$ инъективна, поэтому определения корректны. Кроме того, нетрудно проверить, что $g_{2} \circ g_{1}: x \rightarrow f(x) \rightarrow v_{B}(x) \rightarrow x, x \in A, f(x) \in C$. Из этого следует, что $g_{1} \circ g_{2} \circ g_{1}: x \leftrightarrow f(x), x \in A, f(x) \in C$. Таким образом, $f_{3}=g_{1} \circ g_{2} \circ g_{1}$, или $f=f_{1} \circ f_{2} \circ g_{1} \circ g_{2} \circ g_{1}$. Заметим, что $f_{1}, f_{2}, g_{1}, g_{2} \in Q$. Кроме того, $f_{1}, g_{1}-$ паросочетания на $A \cup B$, а $f_{2}, g_{2}$ - паросочетания на $B \cup C$. Предложение доказано.

Предложение 16. Пусть класс $Q$ удовлетворяет требованиям I, IV. Кроме того, пусть $\left\{A_{1}, \ldots, A_{n}\right\}$ - разбиение множества $A \subseteq \mathbf{N}$ на регулярные в $Q$ множества, $n \geqslant 2$. Тогда любое паросочетание $f \in Q$ на множестве $A$ можно представить в виде композиции перестановок из $Q$, каждая из которых является паросочетанием на множестве вида $A_{i} \cup A_{i+1}, 1 \leqslant i \leqslant n-1$. 
Доказательство. Докажем это предложение индукцией по $n$. При $n=2$ утверждение очевидно. Пусть $n \geqslant 3$ и утверждение доказано для значений $2, \ldots, n-1$. Применим предложение 15 для множеств $A_{1}, A_{2} \cup \ldots \cup A_{n-1}$ и $A_{n}$ (из предложения 5 следует, что эти множества регулярны). Получаем, что $f$ представима в виде композиции перестановок из $Q$, каждая из которых - паросочетание на множестве $A_{1} \cup \ldots \cup A_{n-1}$ или $A_{2} \cup \ldots \cup A_{n}$. Применив для каждой из этих перестановок и соответствующих множеств предположение индукции, получим доказываемое утверждение.

Предложение 17. Пусть паросочетание $f$ и множество четырехмерных векторов $B$ с различными компонентами (внутри векторов и в разных векторах) удовлетворяют (1). Кроме того, пусть паросочетания $f_{1}^{\prime} u f_{2}^{\prime}$ на множестве $A \subseteq \mathbf{N}$ определяются соотношениями

$$
\begin{aligned}
& f_{1}^{\prime}: \quad b_{1} \leftrightarrow b_{2}, \quad b_{3} \leftrightarrow b_{4}, \quad\left(b_{1}, b_{2}, b_{3}, b_{4}\right) \in B, \\
& f_{2}^{\prime}: b_{1} \leftrightarrow b_{3}, \quad\left(b_{1}, b_{2}, b_{3}, b_{4}\right) \in B,
\end{aligned}
$$

И пусть $f_{1}^{\prime \prime}, f_{2}^{\prime \prime}$ - паросочетания на множествах $A_{1}^{\prime \prime}$ и $A_{2}^{\prime \prime}$ соответственно, $A, A_{1}^{\prime \prime}, A_{2}^{\prime \prime}$ попарно не пересекаются, $f_{1}=f_{1}^{\prime} \circ f_{1}^{\prime \prime}, f_{2}=f_{2}^{\prime} \circ f_{2}^{\prime \prime}$. Тогда $f=\left(f_{1} \circ f_{2}\right)^{2}$.

Доказательство. Отметим, что если $\varphi, \psi-$ паросочетания на множествах $A_{\varphi}$ и $A_{\psi}$ соответственно, $A_{\varphi} \cap A_{\psi}=\varnothing$, то $\varphi \circ \psi=\psi \circ \varphi$. Отсюда и из того, что $A, A_{1}^{\prime \prime}, A_{2}^{\prime \prime}$ попарно не пересекаются, следует, что

$$
\begin{aligned}
\left(f_{1} \circ f_{2}\right)^{2} & =f_{1}^{\prime} \circ f_{1}^{\prime \prime} \circ f_{2}^{\prime} \circ f_{2}^{\prime \prime} \circ f_{1}^{\prime} \circ f_{1}^{\prime \prime} \circ f_{2}^{\prime} \circ f_{2}^{\prime \prime} \\
& =\left(f_{1}^{\prime} \circ f_{2}^{\prime}\right)^{2} \circ\left(f_{1}^{\prime \prime}\right)^{2} \circ\left(f_{2}^{\prime \prime}\right)^{2}=\left(f_{1}^{\prime} \circ f_{2}^{\prime}\right)^{2} .
\end{aligned}
$$

Из (24) и (25) следует, что $f_{1}^{\prime} \circ f_{2}^{\prime}: b_{1} \rightarrow b_{4} \rightarrow b_{3} \rightarrow b_{2} \rightarrow b_{1},\left(b_{1}, b_{2}, b_{3}, b_{4}\right) \in B$. Из этого вытекает, что $\left(f_{1}^{\prime} \circ f_{2}^{\prime}\right)^{2}: b_{1} \leftrightarrow b_{3}, b_{2} \leftrightarrow b_{4},\left(b_{1}, b_{2}, b_{3}, b_{4}\right) \in B$. Правая часть этой формулы совпадает с правой частью (1). Таким образом, $f=\left(f_{1}^{\prime} \circ f_{2}^{\prime}\right)^{2}$. Предложение доказано.

Введем несколько вспомогательных определений. Пусть класс $Q$ удовлетворяет требованиям I-III, V. Тогда по следствию 1 существует конечное число перестановок из $\operatorname{Gr}(Q)$, в виде композиции которых можно представить любую перестановку из $\operatorname{Gr}(Q)$. Из этого и из теоремы 1 следует, что существуют правильные в $Q$ тройки $\left(f_{1}, g_{1}, B_{1}^{\prime}\right), \ldots,\left(f_{n}, g_{n}, B_{n}^{\prime}\right)$ такие, что множество, состоящее из паросочетаний

$$
f_{1}, \ldots, f_{n}
$$

порождает $\operatorname{Gr}(Q)$.

Определим вектор-функцию $\delta: \mathbf{N}^{4} \rightarrow \mathbf{N}^{4}$ равенством

$$
\delta\left(b_{1}, b_{2}, b_{3}, b_{4}\right)= \begin{cases}\left(b_{1}, b_{2}, b_{3}, b_{4}\right), & \text { если } b_{1}<b_{2}, \\ \left(b_{2}, b_{1}, b_{4}, b_{3}\right) & \text { в противном случае. }\end{cases}
$$

Для всех $i, 1 \leqslant i \leqslant n$, положим $B_{i}=\left\{\delta\left(b_{1}, b_{2}, b_{3}, b_{4}\right):\left(b_{1}, b_{2}, b_{3}, b_{4}\right) \in B_{i}^{\prime}\right\}$. Отметим, что $\left(f_{1}, g_{1}, B_{1}\right), \ldots,\left(f_{n}, g_{n}, B_{n}\right)$ - правильные в $Q$ тройки. Кроме того,

$$
B_{i}=\left\{\left(x, g_{i}(x), f_{i}(x), f_{i}\left(g_{i}(x)\right)\right): x<g_{i}(x)\right\}, \quad 1 \leqslant i \leqslant n .
$$


Положим

$$
\begin{aligned}
& h_{i}: \quad b_{1} \leftrightarrow b_{2}, \quad b_{3} \leftrightarrow b_{4}, \quad\left(b_{1}, b_{2}, b_{3}, b_{4}\right) \in B_{i}, \\
& h_{i+n}: \quad b_{1} \leftrightarrow b_{3}, \quad\left(b_{1}, b_{2}, b_{3}, b_{4}\right) \in B_{i}, \quad 1 \leqslant i \leqslant n .
\end{aligned}
$$

С учетом (27) это можно переписать в виде

$$
\begin{aligned}
h_{i}: & x \leftrightarrow g_{i}(x), \quad f_{i}(x) \leftrightarrow f_{i}\left(g_{i}(x)\right), & & x<g_{i}(x), \\
h_{i+n}: & x \leftrightarrow f_{i}(x), & & x<g_{i}(x) .
\end{aligned}
$$

Пусть

$$
\begin{aligned}
\operatorname{rol}(x) & =x-\operatorname{rm}\left(x, 2^{2 n+1}\right)+\operatorname{rm}\left(x+1,2^{2 n+1}\right), \\
E_{0} & =\left\{2^{2 n+1} x, x \in \mathbf{N}\right\}, \\
E_{i} & =\operatorname{rol}^{i}\left(E_{0}\right), \quad 1 \leqslant i<2^{2 n+1} .
\end{aligned}
$$

Отметим, что $\left\{E_{0}, \ldots, E_{2^{2 n+1}-1}\right\}-$ разбиение множества $\mathbf{N}$. Ясно, что $E_{0}$ регулярно в $Q\left(\mu_{E_{0}}(x)=\left[x / 2^{2 n+1}\right]\right.$, если $\operatorname{rm}\left(x, 2^{2 n+1}\right)=0, \mu_{E_{0}}(x)=0$, в противном случае, $\left.v_{E_{0}}(x)=2^{2 n+1} x\right)$, аналогично доказывается регулярность в $Q$ всех множеств $E_{i}$, $1 \leqslant i<2^{2 n+1}$. Из предложения 5 следует, что $E_{0} \cup E_{1}$ регулярно в $Q$.

Положим

$$
\begin{array}{rlr}
u_{i}: & v_{E_{0} \cup E_{1}}(x) \rightarrow v_{E_{0} \cup E_{1}}\left(h_{i}(x)\right), & \\
v_{i}= & \operatorname{rol}^{2^{i}} \circ u_{i} \circ \mathrm{rol}^{-2^{i}}, & 1 \leqslant i \leqslant 2 n, \\
\text { all }= & v_{1} \circ \ldots \circ v_{2 n}, & \\
w_{i}: & v_{E_{0} \cup E_{1}}(x) \rightarrow v_{E_{0} \cup E_{1}}\left(f_{i}(x)\right), & 1 \leqslant i \leqslant n .
\end{array}
$$

Предложение 18. Справедливы соотношения rol, $\mathrm{rol}^{-1}$, all $=\mathrm{all}^{-1} \in Q$.

Доказательство. Утверждение для rol и $\mathrm{rol}^{-1}$ следует непосредственно из (32). Из (30) и (31) следует, что $h_{i} \in Q, 1 \leqslant i \leqslant 2 n$, из этого и из (35), (36), (37) следует, что all $\in Q$. Кроме того, из (30) и (31) следует, что $h_{i}$ - паросочетание при любом $i, 1 \leqslant i \leqslant 2 n$. Из этого и из (35) вытекает, что $u_{i}$ - паросочетание на $E_{0} \cup E_{1}$, из (36) следует, что $v_{i}$ - паросочетание на $E_{2^{i}} \cup E_{2^{i}+1}, 1 \leqslant i \leqslant 2 n$. Таким образом, $v_{1}, \ldots, v_{2 n}-$ паросочетания на попарно непересекающихся множествах. Из этого и из (37) следует, что all паросочетание. Следовательно, all ${ }^{-1}=$ all $\in Q$.

Предложение 19. Пусть $n \geqslant 1,1 \leqslant i \leqslant n$, при всех $j, 1 \leqslant j \leqslant 2 n$, числа $\alpha_{j}$ и $\beta_{j}$ определяются равенствами

$$
\alpha_{j}=\operatorname{rm}\left(2^{2 n+1}+2^{j}-2^{i}, 2^{2 n+1}\right), \quad \beta_{j}=\operatorname{rm}\left(2^{2 n+1}+2^{j}-2^{i+n}, 2^{2 n+1}\right) .
$$

Тогда числа $0,1, \alpha_{j}, \alpha_{j}+1, j \neq i, \beta_{j}, \beta_{j}+1, j \neq i+n$, попарно различны.

Доказательство. С учетом того, что все числа $\alpha_{j}, \beta_{j}$ четные, достаточно доказать, что числа $0, \alpha_{j}, j \neq i, \beta_{j}, j \neq i+n$, попарно различны. Докажем это от противного. Пусть $\alpha_{j}=0$. Тогда $2^{j} \equiv 2^{i}\left(\bmod 2^{2 n+1}\right)$, то есть $i=j$. Аналогично, если $\beta_{j}=0$, то $j=i+n$. Если $\alpha_{j_{1}}=\alpha_{j_{2}}$, то $2^{j_{1}} \equiv 2^{j_{2}}\left(\bmod 2^{2 n+1}\right)$, то есть $j_{1}=j_{2}$. Аналогично разбирается случай $\beta_{j_{1}}=\beta_{j_{2}}$. Если $\alpha_{j_{1}}=\beta_{j_{2}}$, то $2^{j_{1}}+2^{i+n} \equiv 2^{j_{2}}+2^{i}\left(\bmod 2^{2 n+1}\right)$, то есть $2^{j_{1}}+2^{i+n}=2^{j_{2}}+2^{i}$ (потому что левая и правая части лежат между 4 и $2^{2 n+1}$ ). Это равенство возможно, только если $j_{1}=i$ и $j_{2}=i+n$. Предложение доказано. 
Предложение 20. Перестановки $w_{i}, 1 \leqslant i \leqslant n$, представимы в виде композиции перестановок all $u$ rol.

Доказательство. Зафиксируем $i$. Пусть

$$
\begin{aligned}
& s_{1}=\operatorname{rol}^{-2^{i}} \circ \text { all } \circ \mathrm{rol}^{2^{i}}=\operatorname{rol}^{2^{2 n+1}-2^{i}} \circ \text { all } \circ \mathrm{rol}^{2^{i}}, \\
& s_{2}=\operatorname{rol}^{-2^{i+n}} \circ \text { all } \circ \mathrm{rol}^{2^{i+n}}=\operatorname{rol}^{2^{2 n+1}-2^{i+n}} \circ \text { all } \circ \mathrm{rol}^{2^{i+n}} .
\end{aligned}
$$

Справедливы равенства

$$
\begin{aligned}
s_{1} & =\operatorname{rol}^{-2^{i}} \circ v_{1} \circ \ldots \circ v_{2 n} \circ \mathrm{rol}^{2^{i}} \\
& =\left(\operatorname{rol}^{-2^{i}} \circ v_{1} \circ \operatorname{rol}^{2^{i}}\right) \circ \ldots \circ\left(\mathrm{rol}^{-2^{i}} \circ v_{2 n} \circ \mathrm{rol}^{2^{i}}\right) \\
& =p_{1}^{\prime} \circ \ldots \circ p_{2 n}^{\prime},
\end{aligned}
$$

где

$$
p_{j}^{\prime}=\operatorname{rol}^{2^{j}-2^{i}} \circ u_{j} \circ \operatorname{rol}^{2^{i}-2^{j}}, \quad 1 \leqslant j \leqslant 2 n .
$$

Из (32), (34), предложения 8 и того, что $u_{j}$ - паросочетание на $E_{0} \cup E_{1}$, следует, что $p_{j}^{\prime}$ - паросочетание на $E_{\alpha_{j}} \cup E_{\alpha_{j}+1}$, где

$$
\alpha_{j}=\operatorname{rm}\left(2^{2 n+1}+2^{j}-2^{i}, 2^{2 n+1}\right), \quad 1 \leqslant j \leqslant 2 n .
$$

Аналогично,

$$
s_{2}=p_{1}^{\prime \prime} \circ \ldots \circ p_{2 n}^{\prime \prime}
$$

где

$$
p_{j}^{\prime \prime}=\operatorname{rol}^{2^{j}-2^{i+n}} \circ u_{j} \circ \operatorname{rol}^{2^{i+n}-2^{j}}, \quad 1 \leqslant j \leqslant 2 n,
$$

$p_{j}^{\prime \prime}$ - паросочетание на $E_{\beta_{j}} \cup E_{\beta_{j}+1}$, где

$$
\beta_{j}=\operatorname{rm}\left(2^{2 n+1}+2^{j}-2^{i+n}, 2^{2 n+1}\right), \quad 1 \leqslant j \leqslant 2 n .
$$

Из предложения 19 следует, что множество $E_{0} \cup E_{1}$ и все множества $E_{\alpha_{j}} \cup E_{\alpha_{j}+1}, j \neq i$, $E_{\beta_{j}} \cup E_{\beta_{j}+1}, j \neq i+n$, попарно не пересекаются. Отсюда

$$
\begin{aligned}
p_{i}^{\prime} \circ p_{j}^{\prime} & =p_{j}^{\prime} \circ p_{i}^{\prime}, & & j \neq i, \\
p_{i+n}^{\prime \prime} \circ p_{j}^{\prime \prime} & =p_{j}^{\prime \prime} \circ p_{i+n}^{\prime \prime}, & & j \neq i+n .
\end{aligned}
$$

Из этого можно сделать вывод, что

$$
s_{1}=p_{1}^{\prime} \circ \ldots \circ p_{2 n}^{\prime}=s_{1}^{\prime} \circ s_{1}^{\prime \prime},
$$

где

$$
\begin{aligned}
s_{1}^{\prime} & =p_{i}^{\prime}, \\
s_{1}^{\prime \prime} & =p_{1}^{\prime} \circ \ldots \circ p_{i-1}^{\prime} \circ p_{i+1}^{\prime} \circ \ldots \circ p_{2 n}^{\prime} .
\end{aligned}
$$


Аналогично,

$$
s_{2}=s_{2}^{\prime} \circ s_{2}^{\prime \prime}
$$

где

$$
\begin{aligned}
s_{2}^{\prime} & =p_{i+n}^{\prime \prime}, \\
s_{2}^{\prime \prime} & =p_{1}^{\prime \prime} \circ \ldots \circ p_{i+n-1}^{\prime \prime} \circ p_{i+n+1}^{\prime \prime} \circ \ldots \circ p_{2 n}^{\prime \prime} .
\end{aligned}
$$

Кроме того, из попарной непересекаемости указанных множеств следует, что $s_{1}^{\prime \prime}, s_{2}^{\prime \prime}-$ паросочетания.

Из (28), (29), (35) следует, что

$$
\begin{aligned}
u_{i}: & v_{E_{0} \cup E_{1}}\left(b_{1}\right) \leftrightarrow v_{E_{0} \cup E_{1}}\left(b_{2}\right), & & \\
& v_{E_{0} \cup E_{1}}\left(b_{3}\right) \leftrightarrow v_{E_{0} \cup E_{1}}\left(b_{4}\right), & & \left(b_{1}, b_{2}, b_{3}, b_{4}\right) \in B_{i}, \\
u_{i+n}: & v_{E_{0} \cup E_{1}}\left(b_{1}\right) \leftrightarrow v_{E_{0} \cup E_{1}}\left(b_{3}\right), & & \left(b_{1}, b_{2}, b_{3}, b_{4}\right) \in B_{i}, \quad 1 \leqslant i \leqslant n .
\end{aligned}
$$

Из (38) и того, что $\left(f_{i}, g_{i}, B_{i}\right)$ - правильная тройка, следует, что

$$
\begin{aligned}
w_{i}: & v_{E_{0} \cup E_{1}}\left(b_{1}\right) \leftrightarrow v_{E_{0} \cup E_{1}}\left(b_{3}\right), \\
v_{E_{0} \cup E_{1}}\left(b_{2}\right) & \leftrightarrow v_{E_{0} \cup E_{1}}\left(b_{4}\right), \quad\left(b_{1}, b_{2}, b_{3}, b_{4}\right) \in B_{i} .
\end{aligned}
$$

где $1 \leqslant i \leqslant n$. Из (41), (44) следует, что

$$
s_{1}^{\prime}=u_{i}
$$

и из (42), (46) следует, что

$$
s_{2}^{\prime}=u_{i+n}
$$

Заметим, что $s_{1}^{\prime}, s_{2}^{\prime}$ - паросочетания на $E_{0} \cup E_{1}$ (это следует из (35), (50), (51)), $s_{1}^{\prime \prime}-$ паросочетание на $\bigcup_{j \neq i}\left(E_{\alpha_{j}} \cup E_{\alpha_{j}+1}\right), s_{2}^{\prime \prime}-$ на $\bigcup_{j \neq i+n}\left(E_{\beta_{j}} \cup E_{\beta_{j}+1}\right)$, указанные множества не пересекаются. Из (43), (45), (47), (48), (49), (50), (51) следует, что для перестановок $w_{i}, s_{1}, s_{2}, s_{1}^{\prime}, s_{2}^{\prime}, s_{1}^{\prime \prime}, s_{2}^{\prime \prime}$ (вместо $f, f_{1}, f_{2}, f_{1}^{\prime}, f_{2}^{\prime}, f_{1}^{\prime \prime}, f_{2}^{\prime \prime}$ соответственно) и множества $B_{i}^{\prime \prime}=\left\{\left(v_{E_{0} \cup E_{1}}\left(b_{1}\right), v_{E_{0}} \cup E_{1}\left(b_{2}\right), v_{E_{0} \cup E_{1}}\left(b_{3}\right), v_{E_{0} \cup E_{1}}\left(b_{4}\right)\right),\left(b_{1}, b_{2}, b_{3}, b_{4}\right) \in B_{i}\right\}$ выполнены все условия предложения 17. Из этого следует, что $w_{i}=\left(s_{1} \circ s_{2}\right)^{2}$. Заметим, что $s_{1}$ и $s_{2}$ представимы в виде композиции rol и all (см. (39), (40)). Предложение доказано.

Предложение 21. Любое паросочетание на $E_{0} \cup E_{1}$, принадлежащее $Q$, представимо в виде композиции rol $u$ all.

Доказательство. Пусть $f$ - данное паросочетание. Положим

$$
g(x)=\mu_{E_{0} \cup E_{1}} \circ f \circ v_{E_{0} \cup E_{1}}(x) .
$$

Нетрудно видеть, что $g$ - паросочетание, принадлежащее $Q$. Следовательно, существуют $i_{1}, \ldots, i_{k}$ такие, что $1 \leqslant i_{1}, \ldots, i_{k} \leqslant n$ и

$$
g=f_{i_{1}} \circ \ldots \circ f_{i_{k}}
$$


$\left(f_{1}, \ldots, f_{n}-\right.$ паросочетания из (26)). Нетрудно заметить, что

$$
f: \quad v_{E_{0} \cup E_{1}}(x) \rightarrow v_{E_{0} \cup E_{1}}(g(x)) .
$$

Из этого, из (38) и (52) следует, что

$$
f=w_{i_{1}} \circ \ldots \circ w_{i_{k}} .
$$

Отсюда и из предложения 20 вытекает доказываемое утверждение.

Предложение 22. Если $0 \leqslant i<2^{2 n+1}-1, f \in Q$ - паросочетание на $E_{i} \cup E_{i+1}$, то $f$ представимо в виде композиции rol $u$ all.

Доказательство. Пусть

$$
f^{\prime}=\operatorname{rol}^{-i} \circ f \circ \operatorname{rol}^{i} .
$$

Из (32), (33), (34) и предложения 8 следует, что $f^{\prime}-$ паросочетание на $E_{0} \cup E_{1}$. Кроме того, очевидно, что

$$
f=\operatorname{rol}^{i} \circ f^{\prime} \circ \operatorname{rol}^{-i}=\operatorname{rol}^{i} \circ f^{\prime} \circ \operatorname{rol}^{2 n+1}-i
$$

Отсюда и из предложения 21 вытекает доказываемое утверждение.

Теорема 3. Любая перестановка $f \in G r(Q)$ может быть представлена в виде композиции rol $u$ all.

Доказательство. Действительно, из теоремы 1 следует, что $f$ можно представить в виде композиции паросочетаний в $Q$, по предложению 16 каждое такое паросочетание представимо в виде композиции паросочетаний на множествах вида $E_{i} \cup E_{i+1}, 0 \leqslant i<2^{2 n+1}-1$, принадлежащих $Q$, по предложению 22 каждое такое паросочетание представимо в виде композиции rol и all.

\section{4. Конечная порождаемость $G r(\mathrm{FP})$}

Пусть FP - множество всех функций $f\left(x_{1}, \ldots, x_{n}\right)$, вычислимых на машине Тьюринга за полиномиальное время (от длины входа при двоичном кодировании входа и выхода).

Теорема 4. Класс FP удовлетворяет требованиям I-III, V.

Доказательство. Требования I, II для FР легко проверяются. Требование V следует из [5]. Докажем, что выполнено требование III. Пусть $f-$ перестановка, $f, f^{-1} \in$ FР. Выберем функцию $h(x)$ вида $2^{\left[\log _{2}(x+4)\right]^{n}}+2 x, n \geqslant 2$, такую, что для любого $x$ справедливы неравенства $f(x), f^{-1}(x)<h(x)$. Заметим, что $h(x)>x$ при любом $x$ и функция $h(x)-x$ возрастает. Пусть

$$
A_{i}=\left\{x: h^{i}(0) \leqslant x<h^{i+1}(0)\right\}, \quad i \geqslant 0 .
$$

Ясно, что $\left\{A_{i}\right\}-$ разбиение множества N. Пусть

$$
\begin{aligned}
& R_{1}=A_{0} \cup A_{4} \cup A_{8} \cup \ldots, \\
& R_{2}=A_{2} \cup A_{6} \cup A_{10} \cup \ldots
\end{aligned}
$$


Если $x \in A_{i}, f(x) \in A_{j}$, то $x<h^{i+1}(0)$ и, следовательно, $f(x)<h^{i+2}(0)$, то есть $j \leqslant i+1$. Аналогично, заметив, что $x=f^{-1}(f(x))$, получаем, что $i \leqslant j+1$, то есть $|i-j| \leqslant 1$. Из этого следует, что

$$
f\left(R_{1}\right) \cap R_{2}=\varnothing
$$

Докажем регулярность $R_{1}$ в FP, регулярность $R_{2}, \mathbf{N} \backslash R_{1}, \mathbf{N} \backslash R_{2}$ доказывается аналогично. Пусть

$$
\mu_{1}(x)= \begin{cases}\text { номер } x \text { в } R_{1} \text { (нумерация по возрастанию с нуля), } & \text { если } x \in R_{1}, \\ 0 & \text { в противном случае }\end{cases}
$$

и пусть $v_{1}(x)$ равно элементу множества $R_{1}$ с номером $x$.

Заметим, что $h(x)>2 x$ при всех $x$, поэтому для вычисления значений $\mu_{1}(x)$ и $\chi_{R_{1}}(x)$ достаточно $\left[\log _{2} x\right]+1$ итераций функции $h$. Из этого, очевидно, следует, что $\mu_{1}, \chi_{R_{1}} \in \mathrm{FP}$. Осталось доказать, что $v_{1} \in \mathrm{FP}$ (для этого, очевидно, достаточно доказать, что она ограничена сверху некоторой функцией из FР).

Отметим, что $A_{0}, A_{1}, \ldots$ - непересекающиеся отрезки в $\mathbf{N}$, их длины $\left|A_{i}\right|$ возрастают при увеличении $i$ (потому что $h(x)-x$ возрастает). Отсюда и из (53) следует, что

$$
\mu_{1}\left(h^{4 i+1}(0)-1\right)+1 \geqslant \frac{h^{4 i+1}(0)}{4}
$$

для любого $i$. Из этого следует, что если $v_{1}(x)=h^{4 i+1}(0)-1$ при некоторых $x$ и $i$, то

$$
v_{1}(x) \leqslant 4 x+3
$$

Докажем, что для любого $x$ справедливо неравенство

$$
v_{1}(x)<h^{5}(4 x+3) \text {. }
$$

Действительно, при $x<h(0)-1$ это очевидно, при $x \geqslant h(0)-1$ выберем наибольшее $i$ такое, что $h^{4 i+1}(0)-1 \leqslant v_{1}(x)$. Пусть

$$
h^{4 i+1}(0)-1=v_{1}\left(x^{\prime}\right) .
$$

Тогда

$$
\begin{aligned}
v_{1}(x) & <h^{4 i+5}(0)-1 \leqslant h^{5}\left(h^{4 i+1}(0)-1\right) \\
& =h^{5}\left(v_{1}\left(x^{\prime}\right)\right) \leqslant h^{5}\left(4 x^{\prime}+3\right) \leqslant h^{5}(4 x+3) .
\end{aligned}
$$

Из этого неравенства следует, что $v_{1}(x) \in \mathrm{FP}$. Таким образом, $f, R_{1}$ и $R_{2}$ удовлетворяют всем условиям из требования III. Теорема доказана.

Следствие 2. $B$ Gr(FP) существуют две перестановки, композициями которых можно представить любую перестановку из $\mathrm{Gr}(\mathrm{FP})$.

Замечание 1. Теорема 4 допускает обобщение на классы Гжегорчика $\mathscr{E} n, n \geqslant 2$ (см. [1]), произвольные $\mathscr{E}^{2}$-замкнутые классы функций, имеющие конечный базис по суперпозиции ([3]) и многие другие классы. Для каждого такого класса нужно соответствующим образом подобрать семейство функций $h$. 


\section{Список литературы}

1. Гжегорчик А., Некоторые классы рекурсивных функций. В сб.: Проблемы математической логики. Мир, Москва, 1970, с. 9-49.

2. Мальцев А. И., Алгоритмы и рекурсивныле функции. Наука, Москва, 1986.

3. Марченков С. С., Базисы по суперпозиции в классах рекурсивных функций. Maтем. вопросы кибернетики (1991) 3, 115-139.

4. Марченков С. С., Элементарные рекурсивные функции. МЦНМО, Москва, 2003.

5. Мучник А. А., О двух подходах к классификации рекурсивных функций. В сб.: Проблемы математической логики. Мир, Москва, 1970, с. 123-138.

Статья поступила 22.06.2007. 